\title{
Clostridioides difficile in Non-hospital Sources (Animals, Food, and Environment) in Asian Countries: A Literature Review
}

\author{
Zahra Esfandiari (iD ${ }^{1,}$, , Fatemeh Amani (iD ${ }^{1}$, Shokofeh Khavari (iD ${ }^{1}$ and Masoud Sami (iD ${ }^{1}$ \\ ${ }^{1}$ Student Research Committee, Department of Food Science and Technology, School of Nutrition and Food Science, Isfahan University of Medical Sciences, Isfahan, Iran \\ "Corresponding author: Student Research Committee, Department of Food Science and Technology, School of Nutrition and Food Science, Isfahan University of Medical \\ Sciences, Isfahan, Iran. Email: z.esfandiari@nutr.mui.ac.ir
}

Received 2021 April 27; Revised 2021 June 12; Accepted 2021 June 13.

\begin{abstract}
Context: Clostridioides difficile (C. difficile) is an agent responsible for severe infection with a high mortality rate in healthcare facilities. With the discovery of $C$. difficile in the community, it was assumed that this bacterium might be transmitted to humans through non-hospital sources.

Evidence Acquisition: This study examined different aspects of the epidemiology of C. difficile in Asian countries with a review of the literature using search engines such as Web of Science, Scopus, and PubMed.

Results: Based on the literature pertaining to Asia, the highest rate of $C$. difficile is found in samples collected from farm animals, red meat, and meat-based products. Two ribotypes 027 and 078, as hypervirulent factors, were found in different non-hospital sources. Resistance to the most frequently used antibiotics in healthcare setting was observed in C. difficile.

Conclusions: Due to the heterogeneity of the examination of $C$. difficile, understanding the actual condition of $C$. difficile is difficult. However, the presence of two hypervirulent ribotypes of $C$. difficile in non-hospital sources is alarming. It seems that it is necessary to perform further studies on C. difficile in non-hospital sources. Defining a focal point for such research could be helpful to explore the situation of $C$. difficile in clinical settings and communities of Asian countries.
\end{abstract}

Keywords: Clostridioides difficile, Non-hospital Sources, Asian Countries

\section{Context}

Clostridioides difficile (C. difficile) is a Gram-positive, toxigenic, and obligate anaerobic bacterium with the capacity for spore development $(1,2)$. Today, $C$. difficile is known as the leading cause of nosocomial infections (CDI), responsible for antibiotic-associated diarrhea (AAD) and pseudomembranous colitis (PMC) in vulnerable and older adults (3). Since C. difficile naturally exists in humans' and animals' digestive systems and intestines, the destruction of the normal enteric flora by antibiotics leads to the organism's growth and toxin production (4). Clostridioides difficile produces two types of toxins $\mathrm{A}(\mathrm{tcdA})$ and $\mathrm{B}(\mathrm{tcdB})$. Nearly all toxigenic forms of $C$. difficile release toxins, and a few clinically relevant strains secrete only toxin B in a colonic environment. Another toxin was discovered in some $C$. difficile strains, named binary toxin (cdt), associated with intensive symptoms $(5,6)$. Significant mortality related to the virulence properties of PCR ribotypes 027 and 078 of $C$. difficile has been highlighted in clinical epidemiology (7).

It is estimated that 453,000 and 172,000 infections occur annually in the United States and Europe. In a study conducted in Asia in 2017, it was estimated that 4,343 out of 37,633 infected people were at CDI risk (8). The epidemiology of CDI has been changing since 2000. The studies show that virulence factors are not connected to traditional risk factors such as antibiotic therapy, previous hospitalization, and advanced age. The genome comparison of $C$. difficile shows that a large proportion of CDI originates from the community (CACDI) (9). The high prevalence of CACDI has raised many questions about the organism's origin. The gene sequence of human $C$. difficile has also been detected in strains isolated from different nonhospital sources, but there is currently no objective evidence of this source of human transmission (10). To better understand CACDI, it would be interesting to examine the studies related to its occurrence. The prevalence of C. difficile varies between different parts of the community; however, there is a lack of any review on this subject in Asian countries. Thus, the present literature review aimed to fill the gap by evaluating the epidemiology of $C$. difficile in different non-hospital sources. 


\section{Evidence Acquisition}

In light of the reports on the $C$. difficile prevalence in non-hospital sources, the current study was conducted to investigate this issue with keywords including prevalence, toxigenicity, ribotyping, and antibiotic resistance in Asian countries using search engines such as Web of Science, Scopus, and Medline from 2010 to 2021.

\section{Results}

\subsection{Farm Animals}

The first study related to farm animals was from Japan in Asia, finding that two out of 250 (0.8\%) fecal samples of pigs were contaminated with $C$. difficile. One strain was positive for the tcdB gene, and the other was negative for the tcdA and tcdB genes (11). Due to public health concerns about $C$. difficile colonization in the pig intestine, another study was conducted on 120 fecal samples from piglets in Japan. A total of 100 C. difficile strains were isolated from 69 (57.5\%) feces, and 61 (61\%) isolates were toxin-positive. Ribotype 078 identified in 12 isolates was genetically related to both humans and pigs (12). Besides, C. difficile was found in 90 out of 150 (60\%) stool samples collected from calves in Iran. The result of this study indicated a high incidence of $C$. difficile with high antibiotic resistance to clindamycin (13). In another Iranian study performed on 150 fecal samples collected from camels, goats, sheep, and cows, the contamination rate of toxigenic C. difficile was $4 \%$ (14). Hussain et al., in a study conducted in India, reported the contamination with $C$. difficile in fecal samples collected from cattle, pigs, and poultry at $4.8 \%, 12.4 \%$, and $13.9 \%$, respectively (15).

A study by Jafari et al. showed that $10 \%$ of 100 fecal samples collected from ostrich had C. difficile, with 70\% toxicity in Iran (16). In another Iranian survey, the prevalence rate of $C$. difficile was found to be 14 out of 40 (35\%) fecal samples collected from chicken. A total of five out of 14 samples were positive for tcdA, tcdB, and cdt genes, and none was positive for binary toxins in molecular identification (17). In Korea, 910 fecal samples from pigs (diarrheic and healthy) were analyzed for $C$. difficile prevalence, and contamination was observed in 98 (30.4\%) diarrheic and 78 (13.3\%) healthy samples. Ribotype 078 identified in most isolates indicated a leading causative pathogen of neonatal diarrhea in piglets (18). The results for 398, 121, and 19 rectal samples from pigs, chickens, and ducks showed that $8.2 \%$ of the samples were contaminated with $C$. difficile, with a toxigenic rate of $88.6 \%$. In this study, the isolation of hypervirulent strain ribotype 078 from piglets was reported for the first time in China (19).

\subsection{Slaughterhouse}

In a study conducted in Iran, $C$. difficile was studied in farm animals from slaughterhouse to retail stage through examining 450 samples of feces, post-eviscerated animals, washed carcasses, and the meat of camels, goats, sheep, and cows in a slaughterhouse, as well as examining 300 samples of chopped and ground meat in the retail stage. Clostridioides difficile was detected in all samples of different sections of the slaughterhouse, which were found to be 20 (13.3\%), 23 (15.3\%), and 11 (7.3\%) for feces, post-eviscerated animals, and washed carcasses, respectively. A total of 79 (26.3\%) samples were contaminated with $C$. difficile in the retail stage, with a higher rate in the ground form $(9.3 \%)$ than in the chopped form (2\%). Toxigenic isolates were found in 29 (3.8\%) samples with 21 different ribotypes specified in Iran (14). The examination of $C$. difficile was reported in 659 colonic content of pigs at local slaughterhouses of six Korean provinces. The prevalence rate was low, and multidrug-resistant $C$. difficile ribotype 078 was present in two (0.3\%) samples (20).

In 422 swab samples from different parts of a pig slaughterhouse, including hiding, scalding water, stool, colon, belly, and carcass surfaces, in Taiwan, toxigenic C. difficile was found in all slaughterhouse sections in high prevalence (48\%), and ribotype 126 was dominant. These ribotypes detected from the slaughterhouse, pig stool, colons, carcasses, and scalding water were closely genetically related, directing serious hazards for crosscontamination (21). To find hypervirulence ribotypes 027 and 078 of $C$. difficile in the slaughter line of Turkey, it was examined 555 final carcasses of cattle and sheep destined both for meat production and retail outlets. The bacterium was detected in $83(33.6 \%)$ cattle and 78 (25.3\%) sheep carcass samples. Ribotype 027 was found in $18.1 \%$ and $7.7 \%$ of the isolates from cattle and sheep, whereas the other hypervirulent isolate ribotype 078 could not be detected among the analyzed samples (22).

\subsection{Meat from Poultry and Birds}

The first report of contamination by $C$. difficile in white meat in Asian countries originated from Iran by Hassanzadeh and Rahimi in 2013. In this study, 240 samples of ostrich and turkey were examined with the isolation of $C$. difficile from 11 (9.1\%) and 14 (11.6\%) samples of ostrich and turkey meat (23). In another study conducted by these researchers, C. difficile was found in 19 out of 120 (15.8\%) chicken samples (24). In a study in Turkey (2015) on 310 different chicken parts, the prevalence rate of toxigenic isolates was low (1.6\%) (25). Another study performed on 65 samples of necks, thighs, and wings in packed chicken in Iran showed that seven (70\%) out of 10 (15.3\%) C. difficile isolates were toxigenic (17). Clostridioides difficile was detected in 25 of 149 (16.8\%) chicken samples evaluated in Korea, and $4.4 \%$ of positive isolates were toxigenic (26). In contrast, C. difficile was not found in 27 samples of chicken breast in Turkey (27). Two parts of chickens, including meat (89) and liver (28), were evaluated for the presence of $C$. difficile in 
the retail stage in Japan. One toxigenic isolate was found in this study and four PCR ribotypes, including F1, F7, F8, and F9, from seven positive isolates of C. difficile (28).

\subsection{Red Meat}

The prevalence of $C$. difficile in raw meat in the retail stage was reported from Iran among 660 samples of red meat. Overall, 13 (2\%) samples were contaminated with $C$. difficile, which mainly belonged to buffalo (9\%), and seven out of those 13 (53.9\%) were toxigenic. Among the positive isolates, seven were ribotype 078 (29). Esfandiari et al. in 2014 found C. difficile in eight (4\%) of 200 samples of chopped and ground beef and mutton meat collected from meat-packaging plants in Iran (5). In addition, these researchers collected a total of 100 beef samples, including 50 chopped and 50 ground, from butcheries. Clostridioides difficile was isolated from 12 (12\%) samples. In both studies, the prevalence rate was higher in ground meat than in chopped meat, suggesting a wide distribution of C. difficile spores in the environment, especially from the meat grinder, due to the formation of a biofilm (30). In contrast, a high prevalence rate was reported in 100 (30\%) samples of ground beef collected from butcheries in Iran (31). In an examination conducted in Turkey on a total of 100 samples, including "modified atmosphere packaging" minced (50) and cubed beef (50), 3\% of samples were contaminated with toxigenic C. difficile (32).

A sampling of 62 raw meat samples, including skin pork and ground pork, was performed in Taiwan. A total of four samples out of 23 (17\%) isolates of $C$. difficile harbored tcdA, tcdB, cdtA, and cdtB (21). Among 266 samples of beef and pork meat examined in Korea, nine (6.8\%) and 11 (8.3\%), respectively, were found positive for C. difficile (26). Lack of C. difficile was reported in 31 ground beef samples in Turkey (27). In Saudi Arabia, of 600 samples of raw cow, sheep, and goat meat, $C$. difficile was discovered in nine (1.5\%) samples. All isolates were toxigenic with a profile of ribotype 078 (33). A collection of 351 samples, including pork meat, pig liver, and beef meat, was examined in Japan. A total of three positive $C$. difficile isolates was found in these samples with two distinct ribotypes belonging to Japan (F10 and R6) (28).

\subsection{Meat-based Products}

In 2014, 211 hamburger, their raw ingredients, and environmental samples were collected from processing plants of Iran. Clostridioides difficile was not detected in 45 non-meat ingredients, including defrosted onions, textured soy proteins, and seasonings. On the other hand, the bacterium was isolated from nine samples, including raw meat (5.6\%), swabs taken from the environment (3.5\%), and hamburger samples (7.1\%) before and after molding (34). In another study conducted by Iranian researchers, 570 meat-based samples, including hamburgers, minced meat, chicken nuggets, sausages, and canned meat, were studied in 2015. Clostridioides difficile was observed in six (1.2\%) samples, of which five were toxigenic isolates: one hamburger and four minced meat samples (35). Some meat-based products were examined for the presence of C. difficile in Turkey. A total of two (4.6\%) samples, including meatball and cooked meat döner, had C. difficile. The method of slow rotated cooking of döner in front of heating elements, and the lack of cooking for meatballs were suggested as the reasons for C. difficile presence (27). In another study conducted in Turkey, 319 meat products were obtained from butcheries and supermarkets to survey $C$. difficile. The toxigenic form of this organism was isolated in $22(6.9 \%)$ examined meat product samples, and nine (40.9\%) isolates were identified as ribotype 027 (36).

\subsection{Ready-to-eat Food}

Iranian researchers examined a variety of 368 samples of unpacked ready-to-eat food. Clostridioides difficile was detected in five (1.36\%) samples, out of which four toxigenic isolates had positive tcdA and tcdB genes (37). Furthermore, 106 samples of ready-to-eat salads were examined for the presence of $C$. difficile in Iran. In this study, six samples (5.66\%) were contaminated with C. difficile (38). A total of 60 samples of ready-to-eat vegetable salads were collected in Iran. The results of this study showed that eight(13.3\%) samples were contaminated with C. difficile (39). A total of 65 ready-to-eat food products, including 35 braised skin and 30 braised colon of pork samples, were collected in the retail stage in Taiwan. Clostridioides difficile was found in 15 samples, out of which three isolates were positive for the tcdA and tcdB genes in the braised colon. None of the positive isolates belonged to ribotype 078 (21).

\subsection{Milk}

The only study on C. difficile in milk was carried out in Iran on 430 raw milk samples from bovine, ovine, caprine, buffalo, and camel. Of the two samples of bovine milk (135) contaminated with C. difficile (1.43\%), isolates obtained from one of these samples harbored the tcdA and tcdB genes with ribotype 078 (40).

\subsection{Molluscan Shellfish}

The existence of $C$. difficile in different natural settings demonstrates its ubiquity. Seafood, mainly edible shellfish, recognized for their capacity to accumulate various pathogens from water through feeding, has been revealed to contain C. difficile. A total of 820 shellfish samples, including oysters, mussels, cockles, and clams, were randomly collected from local harbors and retail centers of Bushehr province, Iran. Toxigenic C. difficile was isolated from 26 (3.17\%) samples (41). 


\subsection{Vegetables}

Contamination of vegetables could be related to irrigation or washing with contaminated water, as well as spore transfer via fertilizers. In Saudi Arabia, out of 200 vegetable samples, including potato and parsnip, three samples were contaminated with $C$. difficile, which were toxigenic with ribotype 078 profile (42). In a study from Japan, C. difficile was isolated from eight (3.3\%) of 242 retail fresh vegetables and fruits. A total of seven different PCR ribotypes of $C$. difficile were found in this study. Positive isolates with ribotype 014 were found worldwide, as well as in Japanese clinical cases (28). In light of the presence of ribotype 078, following good agricultural practices is paramount to help prevent spores from entering the food chain from farm to fork.

\subsection{Household Animals}

Extensive reports from veterinary clinics indicate an increase in the prevalence of $C$. difficile AAD in pets in American countries. A few studies in Asia also reported the prevalence of $C$. difficile in pets. The first study in Asia was performed in India, which examined 117 feces from pups and adult dogs in two groups with and without antibiotic treatment. The results showed that $C$. difficile was present in 16 (13.67\%) isolates collected from adult dogs and pups. Out of these isolates, 10 (62.5\%) were toxigenic. A significant association was found between the rate of isolation of $C$. diffcile and antibiotic use. Different ribotypes, including 012, 014, 046, 010, SLO 131, ACD 001, ACD 002, and ACD 003, were identified in this study (43). According to the only investigation carried out in Iran, the prevalence rate of $C$. difficile in 151 fecal samples obtained from the dog population was $7.9 \%$. The genes tcdA and tcdB profiles were observed in eight of the positive isolates (44). An examination of 204 canine fecal samples conducted in Japan showed a prevalence rate of toxigenic isolates of $47 \%$, as well as 29 different PCR ribotypes (45). The distinguished ribotypes (ST-3, ST-15, and ST129) of C. difficile were detected in 146 and 29 feces of dogs and cats in a study performed in China (46).

\subsection{Food Retail Fomites}

Cross-contamination of food during purchase could be caused by transportation. A study was conducted on 800 samples collected from baskets, trolleys, conveyors, and outgoing shopper's plastic bag surfaces in Saudi Arabia. A total of 12 toxigenic isolates were observed with distinct ribotypes belonging to V, 027, A2, BT1, T1, and BT1 (47). The detection of ribotype 027 in this study may indicate that cleaning the surface is necessary to control the spread of CACDI.

\subsection{Wastewater Treatment Plants}

Water sources are also considered a route of $C$. difficile. Ninety-five samples were collected from a conventional activated sludge treatment plant and a waste stabilization pond system in Iran to examine this possibility. A total of three (13.6\%) samples of digested sludge (22) and two (5\%) samples from waste stabilization (40) ponds were found to be contaminated with toxigenic $C$. difficile harboring the tcdB gene (48).

\subsection{Manure Compost}

In Japan, a total of 14 manure compost samples were collected from pig farms to examine the prevalence of $C$. difficile. It was found that 11 C. difficile strains (82\%) were toxigenic, with ribotype 078 being the most dominant. This study shows that the application of composted manure in agricultural land can pose a serious threat concerning $C$. difficile transmission to the food chain (49).

\section{Conclusions}

Research carried out in different Asian countries has shown different rates of the prevalence of C. difficile, variable ribotypes, and resistance to diverse antibiotics (Table 1) in samples collected from non-hospital sources. Most studies have focused on farm animals and meat, but $C$. difficile has also been evaluated in an extensive range of different non-hospital sources, with the prevalence rate from 1.43 to $88.6 \%$. The highest rate belongs to samples obtained from farm animals, red meat, and meat-based products. Hence, the animals and their derivative products may be considerable source for conveying C. difficile spores to humans.

Based on the finding of the two hypervirulent ribotypes 027 and 078 in different sources, it seems that a molecular relationship exists between clinical and community isolates. This situation increases the awareness about the potential of $C$. difficile as an agent for CACDI. Therefore, it is required to implement preventive measures and certain hygiene management activities to control C. difficile in Asian countries.

Most $C$. difficile isolates obtained from different samples were resistant to commonly used antimicrobial agents in healthcare settings, demonstrating the importance of ongoing long-term surveillance of antimicrobial resistance. Due to limitations in the size of sampling and heterogeneity of examination, it is difficult to interpret the exact prevalence of $C$. difficile. In sum, it is required to define a focal reference for clinical and non-hospital isolates of $C$. difficile to help further research on the relationship between those sources. 


\begin{tabular}{|c|c|c|}
\hline Sample & Resistance & Reference \\
\hline Feces of piglet & Ceftriaxone ciprofloxacin, clindamycin, erythromycin & $(12)$ \\
\hline Feces of calf & Ciprofloxacin clindamycin, erythromycin, tetracycline, vancomycin & (13) \\
\hline Slaughtered pigs & Cefoxitin ciprofloxacin, clindamycin erythromycin, moxifloxacin & (20) \\
\hline Sheep and cattle carcass & Cefotaxime, imipenem & $(22)$ \\
\hline Raw beef, cow, sheep, goat, camel and buffalo meat & $\begin{array}{c}\text { Ampicillin, ciprofloxacin, clindamycin, erythromycin, gentamycin, } \\
\text { nalidixic acid, tetracyclin }\end{array}$ & $(29)$ \\
\hline Packaged minced and cubed beef meat & Clindamycin & $(32)$ \\
\hline Raw meat & Clindamycin & $(26)$ \\
\hline Meatball and meat doner & Clindamycin, metronidazole, moxifloxacin tetracycline, vancomycin & $(27)$ \\
\hline Meat products & Cefotaxime, imipenem & $(36)$ \\
\hline $\begin{array}{l}\text { Salad (fasl, maccaroni, and olovyeh), falafel sandwich, and yoghurt } \\
\text { stew }\end{array}$ & $\begin{array}{c}\text { Ampicillin, ciprofloxacin, clindamycin, erythromycin, gentamicin, } \\
\text { nalidixic acid, tetracycline }\end{array}$ & (37) \\
\hline Feces of canine & $\begin{array}{l}\text { Ceftriaxone, ciprofloxacin, clindamycin, erythromycin, levofloxacin, } \\
\text { sitafloxacin }\end{array}$ & $(45)$ \\
\hline Feces of dog and cat & Ampicillin, Cefoxitin, Clindamycin, Vancomycin & $(46)$ \\
\hline Manure compost of pigs & Ciprofloxacin, clindamycin, erythromycin, levofloxacin, tetracycline & $(49)$ \\
\hline Rectal swabs from pigs, chickens and ducks & $\begin{array}{l}\text { Amoxicillin-clavulanic acid, ampicillin, cefotaxime, cefoxitin, } \\
\text { ciprofloxacin, clindamycin, chloramphenicol, erythromycin, imipenem, } \\
\text { meropenem, moxifloxacin, tetracycline }\end{array}$ & (19) \\
\hline Ready to-eat vegetable salads & Clindamycin, nalidixic acid & $(39)$ \\
\hline Raw bovine, ovine, caprine, camel and buffalo milk & Ampicillin, clindamycin, gentamicin, nalidixic acid & $(40)$ \\
\hline Shellfish & Amoxicillin, ampicillin, metronidazole, moxifloxacin, penicillin & (41) \\
\hline Vegetables & Cefotaxime & $(42)$ \\
\hline Retail fomites & Levofloxacin & $(47)$ \\
\hline
\end{tabular}

\section{Footnotes}

Authors' Contribution: Zahra Esfandiari: Study concept and design, acquisition of data, analysis, and interpretation of data, drafting of the manuscript, critical revision of the manuscript for important intellectual content, statistical analysis, administrative, technical, and material support, and study supervision. Fatemeh Amani: Study concept and design, acquisition of data, analysis and interpretation of data, and drafting of the manuscript. Shokofeh Khavari: Study concept and design, acquisition of data, analysis and interpretation of data, and drafting of the manuscript. Masoud Sami: study concept.

Conflict of Interests: None.

Funding/Support: This research is approved in "Student Research Committee" of "School of Nutrition and Food Science" in "Isfahan University of Medical Sciences" with no. 198189. The authors appreciate "Chancellery of Research and Technology" of "Isfahan University of Medical Sciences" for financial supports.

\section{References}

1. Lawson PA, Citron DM, Tyrrell KL, Finegold SM. Reclassifica- tion of clostridium difficile as clostridioides difficile (Hall And O'toole 1935) prevot 1938. Anaerobe. 2016;40:95-9. doi: 10.1016/j.anaerobe.2016.06.008. [PubMed: 27370902].

2. Heise J, Witt P, Maneck C, Wichmann-Schauer H, Maurischat S. Prevalence and phylogenetic relationship of Clostridioides difficile strains in fresh poultry meat samples processed in different cutting plants. Int J Food Microbiol. 2021;339:109032. doi: 10.1016/j.ijfoodmicro.2020.109032. [PubMed: 33388709].

3. Brown KA, Langford B, Schwartz KL, Diong C, Garber G, Daneman $\mathrm{N}$. Antibiotic prescribing choices and their comparative c. Difficile infection risks: A longitudinal case-cohort study. Clin Infect Dis. 2021;72(5):836-44. doi: 10.1093/cid/ciaa124. [PubMed: 32069358]. [PubMed Central: PMC7935390].

4. Patton L, Li N, Garrett TJ, Ruoss JL, Russell JT, de la Cruz D, et al. Antibiotics effects on the fecal metabolome in preterm infants. Metabolites. 2020;10(8). doi: 10.3390/metabo10080331. [PubMed: 32823682]. [PubMed Central: PMC7464203].

5. Esfandiari Z, Jalali M, Ezzatpanah H, Weese JS, Chamani M. Prevalence and characterization of clostridium difficile in beef and mutton meats of Isfahan region, Iran. Jundishapur J Microbiol. 2014;7(8). e16771. doi: 10.5812/jjm.16771. [PubMed: 25485054]. [PubMed Central: PMC4255216].

6. Schwartz R, Guichard A, Franc NC, Roy S, Bier E. A drosophila model for clostridium difficile toxin cdt reveals interactions with multiple effector pathways. iScience. 2020;23(2):100865. doi: 10.1016/j.isci.2020.100865. [PubMed: 32058973]. [PubMed Central: PMC7011083]. 
7. Weese JS. Clostridium difficile in food-innocent bystander or serious threat? Clin Microbiol Infect. 2010;16(1):3-10. doi: 10.1111/j.14690691.2009.03108.x. [PubMed: 20002685].

8. Borren NZ, Ghadermarzi S, Hutfless S, Ananthakrishnan AN. The emergence of Clostridium difficile infection in Asia: A systematic review and meta-analysis of incidence and impact. PLoS One. 2017;12(5). e0176797. doi: 10.1371/journal.pone.0176797. [PubMed: 28463987]. [PubMed Central: PMC5413003].

9. Candel-Perez C, Ros-Berruezo G, Martinez-Gracia C. A review of Clostridioides [Clostridium] difficile occurrence through the food chain. Food Microbiol. 2019;77:118-29. doi: 10.1016/j.fm.2018.08.012. [PubMed: 30297042].

10. RudnickW, Science M, Thirion DJG, Abdesselam K, Choi KB, Pelude L, et al. Antimicrobial use among adult inpatients at hospital sites within the Canadian Nosocomial Infection Surveillance Program: 2009 to 2016. Antimicrob Resist Infect Control. 2020;9(1):32. doi: 10.1186/s13756020-0684-2. [PubMed: 32054539]. [PubMed Central: PMC7020554].

11. Asai T, Usui M, Hiki M, Kawanishi M, Nagai H, Sasaki Y. Clostridium difficile isolated from the fecal contents of swine in Japan. J Vet Med Sci. 2013;75(4):539-41. doi: 10.1292/jvms.12-0353. [PubMed: 23171688].

12. Usui M, Nanbu Y, Oka K, Takahashi M, Inamatsu T, Asai T, et al. Genetic relatedness between Japanese and European isolates of Clostridium difficile originating from piglets and their risk associated with human health. Front Microbiol. 2014;5:513. doi: 10.3389/fmicb.2014.00513. [PubMed: 25339943]. [PubMed Central: PMC4189341].

13. Doosti A, Mokhtari-Farsani A. Study of the frequency of Clostridium difficile tcdA, tcdB, cdtA and cdtB genes in feces of Calves in south west of Iran. Ann Clin Microbiol Antimicrob. 2014;13:21. doi: 10.1186/1476-071113-21. [PubMed: 24903619]. [PubMed Central: PMC4060091].

14. Esfandiari Z, Weese JS, Ezzatpanah H, Chamani M, Shoaei P, Yaran $\mathrm{M}$, et al. Isolation and characterization of Clostridium difficile in farm animals from slaughterhouse to retail stage in Isfahan, Iran. Foodborne Pathog Dis. 2015;12(10):864-6. doi: 10.1089/fpd.2014.1910. [PubMed: 26440207].

15. Hussain I, Borah P, Sharma RK, Rajkhowa S, Rupnik M, Saikia DP, et al. Molecular characteristics of Clostridium difficile isolates from human and animals in the North Eastern region of India. Mol Cell Probes. 2016;30(5):306-11. doi: 10.1016/j.mcp.2016.08.010. [PubMed: 27569848].

16. Jafari SM, Kalidari G, Peighambari SM, Razmyar J. Ostrich as a possible source of pathogenic strains of Clostridium difficile. Thai J Vet Med. 2017;47(4):537-41.

17. Razmyar J, Jamshidi A, Khanzadi S, Kalidari G. Toxigenic Clostridium difficile in retail packed chicken meat and broiler flocks in northeastern Iran. Iran J Vet Res. 2017;18(4):271-4. [PubMed: 29387100]. [PubMed Central: PMC5767634].

18. Kim HY, Cho A, Kim JW, Kim H, Kim B. High prevalence of Clostridium difficile PCR ribotype 078 in pigs in Korea.Anaerobe. 2018;51:42-6. doi: 10.1016/j.anaerobe.2018.03.012. [PubMed: 29604338].

19. Zhang LJ, Yang L, Gu XX, Chen PX, Fu JL, Jiang HX. The first isolation of Clostridium difficile RT078/ST11 from pigs in China. PLoS One. 2019;14(2). e0212965. doi: 10.1371/journal.pone.0212965. [PubMed: 30807599]. [PubMed Central: PMC6391006].

20. Cho A, Byun JW, Kim JW, Oh SI, Lee MH, Kim HY. Low prevalence of clostridium difficile in slaughter pigs in Korea. J Food Prot. 2015;78(5):1034-6. doi: 10.4315/0362-028X.JFP-14-493. [PubMed: 25951403].

21. Wu YC, Chen CM, Kuo CJ, Lee JJ, Chen PC, Chang YC, et al. Prevalence and molecular characterization of Clostridium difficile isolates from a pig slaughterhouse, pork, and humans in Taiwan. Int J Food Microbiol. 2017;242:37-44. doi: 10.1016/j.ijfoodmicro.2016.11.010. [PubMed: 27870984].

22. Hampikyan H, Bingol EB, Muratoglu K, Akkaya E, Cetin O, Colak H. The prevalence of Clostridium difficile in cattle and sheep carcasses and the antibiotic susceptibility of isolates. Meat Sci. 2018;139:120-4. doi: 10.1016/j.meatsci.2018.01.020. [PubMed: 29413671].

23. Hasanzade A, Rahimi E. Isolation of clostridium difficile from Turkey and ostrich meat sold in meat stores if Isfahan city. Int JAdv Biol Biomed Res. 2013;1(9):963-7.
24. Hasanzadeh A, Rahimi E. Isolation of Clostridium difficile from chicken meat sold in meat stores of Isfahan City. Adv Environ Biol. 2013:2372-5.

25. Guran HS, Ilhak OI. Clostridium difficile in retail chicken meat parts and liver in the Eastern Region of Turkey. J Verbrauch Lebensm. 2015;10(4):359-64. doi: 10.1007/s00003-015-0950-z.

26. Lee JY, Lee DY, Cho YS. Prevalence of Clostridium difficile isolated from various raw meats in Korea. Food Sci Biotechnol. 2018;27(3):8839. doi: 10.1007/s10068-018-0318-0. [PubMed: 30263815]. [PubMed Central: PMC6049684].

27. Ersoz SS, Cosansu S. Prevalence of Clostridium difficile Isolated from Beef and Chicken Meat Products in Turkey. Korean J Food Sci Anim Resour. 2018;38(4):759-67. doi: 10.5851/kosfa.2018.e14. [PubMed: 30206435]. [PubMed Central: PMC6131381].

28. Usui M, Maruko A, Harada M, Kawabata F, Sudo T, Noto S, et al. Prevalence and characterization of Clostridioides difficile isolates from retail food products (vegetables and meats) in Japan. Anaerobe. 2020;61:102132. doi: 10.1016/j.anaerobe.2019.102132. [PubMed: 31778810].

29. Rahimi E, Jalali M, Weese JS. Prevalence of Clostridium difficile in raw beef, cow, sheep, goat, camel and buffalo meat in Iran. BMC Public Health. 2014;14:119. doi: 10.1186/1471-2458-14-119. [PubMed: 24499381] [PubMed Central: PMC3918140].

30. Esfandiari Z, Jalali M, Ezzatpanah H, Weese S, Chamani M. Examination of clostridium difficile contamination in beef meat distributed in Isfahan using culture and multiplex-PCR method. BJM. 2014;3(11).

31. Kheradmand M, Jalilian S, Alvandi A, Abiri R. Prevalence of Clostridium difficile and its toxigenic genotype in beef samples in west of Iran. Iran J Microbiol. 2017;9(3):169-73. [PubMed: 29225756]. [PubMed Central: PMC5719511]

32. Atasoy A, GÜCÜKoĞLu A. Detection of Clostridium difficile and toxin genes in samples of modified atmosphere packaged (MAP) minced and cubed beef meat. Ankara Üniv Vet Fak Derg. 2017;64(3):165-70. doi: 10.1501/Vetfak_0000002794.

33. Bakri M. Prevalence of Clostridium difficile in raw cow, sheep, and goat meat in Jazan, Saudi Arabia. Saudi J Biol Sci. 2018;25(4):783-5 doi: 10.1016/j.sjbs.2016.07.002. [PubMed: 29740244]. [PubMed Central: PMC5936870].

34. Esfandiari Z, Weese S, Ezzatpanah H, Jalali M, Chamani M. Occurrence of Clostridium difficile in seasoned hamburgers and seven processing plants in Iran. BMC Microbiol. 2014;14:283. doi: 10.1186/s12866-0140283-6. [PubMed: 25420512]. [PubMed Central: PMC4247873].

35. Rahimi E, Khaksar F. Detection of toxigenic Clostridium difficile strains isolated from meat and meat products in Iran. J Vet Med Sci. 2015;18(3):277-81. doi: 10.15547/bjvm.845.

36. Muratoglu K, Akkaya E, Hampikyan H, Bingol EB, Cetin O, Colak H. Detection, Characterization and Antibiotic Susceptibility of Clostridioides (Clostridium) difficile in Meat Products. Food Sci Anim Resour 2020;40(4):578-87. doi: 10.5851/kosfa.2020.e34. [PubMed: 32734265]. [PubMed Central: PMC7372980].

37. Rahimi E, Afzali ZS, Baghbadorani ZT. Clostridium difficile in readyto-eat foods in Isfahan and Shahrekord, Iran. Asian Pac J Trop Biomed. 2015;5(2):128-31. doi:10.1016/s2221-1691(15)30156-8.

38. Yamoudy M, Mirlohi M, Isfahani BN, Jalali M, Esfandiari Z, Hosseini NS. Isolation of toxigenic Clostridium difficile from ready-to-eat salads by multiplex polymerase chain reaction in Isfahan, Iran. Adv Biomed Res 2015;4:87. doi:10.4103/2277-9175.156650.[PubMed: 26015913].[PubMed Central: PMC4434448]

39. Kochakkhani H, Moosavy MH, Dehghan P. [Isolation and identification of Clostridium difficile from ready-to-eat vegetable salads in restaurants of Tabriz by Real-time PCR and determination of the antibiotic resistance pattern]. Sci J Kurd Univ Med Sci. 2017;22(3):101-12. Persian.

40. Rahimi E, Momtaz H, Hemmati M. Occurrence of clostridium difficile in raw bovine, ovine, caprine, camel and buffalo milk in Iran. Kafkas Univ Vet Fak Derg. 2014. doi: 10.9775/kvfd.2013.10175.

41. Nayebpour F, Rahimi E. Retracted: Prevalence, antibiotic resistance, and toxigenic gene profile of the Clostridium difficile isolated from molluscan shellfish. J Food Saf. 2018;39(1). doi: 10.1111/jfs.12586. 
42. Bakri MM. Investigating the presence of Clostridium difficile in vegetables in Jazan markets, Saudi Arabia. Sky J Microbiol Res. 2016;4(7):60-4.

43. Hussain I, Sharma RK, Borah P, Rajkhowa S, Hussain I, Barkalita $\mathrm{LM}$, et al. Isolation and characterization of Clostridium difficile from pet dogs in Assam, India. Anaerobe. 2015;36:9-13. doi: 10.1016/j.anaerobe.2015.09.006. [PubMed: 26393292].

44. Ghavidel M, Salari Sedigh H, Razmyar J. Isolation of Clostridium difficile and molecular detection of binary and A/B toxins in faeces of dogs. Iran J Vet Res. 2016;17(4):273-6. [PubMed: 28224013]. [PubMed Central: PMC5309461]

45. Usui M, Suzuki K, Oka K, Miyamoto K, Takahashi M, Inamatsu $\mathrm{T}$, et al. Distribution and characterization of Clostridium difficile isolated from dogs in Japan. Anaerobe. 2016;37:58-61. doi: 10.1016/j.anaerobe.2015.10.002. [PubMed: 26456188].

46. Wei Y, Sun M, Zhang Y, Gao J, Kong F, Liu D, et al. Prevalence, geno- type and antimicrobial resistance of Clostridium difficile isolates from healthy pets in Eastern China. BMC Infect Dis. 2019;19(1):46. doi: 10.1186/s12879-019-3678-z. [PubMed: 30634930]. [PubMed Central: PMC6330442].

47. Alqumber MA. Clostridium difficile in retail baskets, trolleys, conveyor belts, and plastic bags in Saudi Arabia. Saudi Med J. 2014;35(10):1274-7. [PubMed: 25316477]. [PubMed Central: PMC4362112].

48. Nikaeen M, Aghili Dehnavi H, Hssanzadeh A, Jalali M. Occurrence of Clostridium difficile in two types of wastewater treatment plants. J Formos Med Assoc. 2015;114(7):663-5. doi: 10.1016/j.jfma.2014.12.005. [PubMed: 25957122].

49. Usui M, Kawakura M, Yoshizawa N, San LL, Nakajima C, Suzuki Y, et al. Survival and prevalence of Clostridium difficile in manure compost derived from pigs. Anaerobe. 2017;43:15-20. doi: 10.1016/j.anaerobe.2016.11.004. [PubMed: 27871997]. 